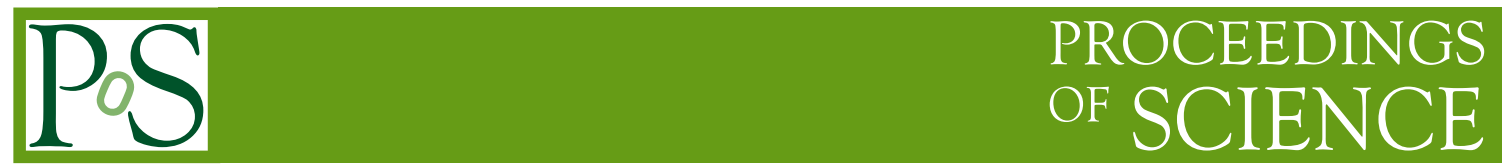

\title{
Higgs boson properties from hadron colliders experiments
}

Pier Paolo GIARDINO*

( Sezione di Pisa (IT))

E-mail: pier.paolo.giardinodpi.infn.it

We perform a state-of-the-art global fit to all Higgs data. We synthesise them into a 'universal' form, which allows to easily test any desired model. We apply the proposed methodology to extract from data the Higgs branching ratios, production cross sections, couplings and to analyse composite Higgs models, extra particles in the loops, invisible Higgs decay into Dark Matter. Best fit regions lie around the Standard Model predictions and are well approximated by our fit.

XXI International Workshop on Deep-Inelastic Scattering and Related Subjects 22-26 April, 2013

Marseilles, France

${ }^{*}$ Speaker. 


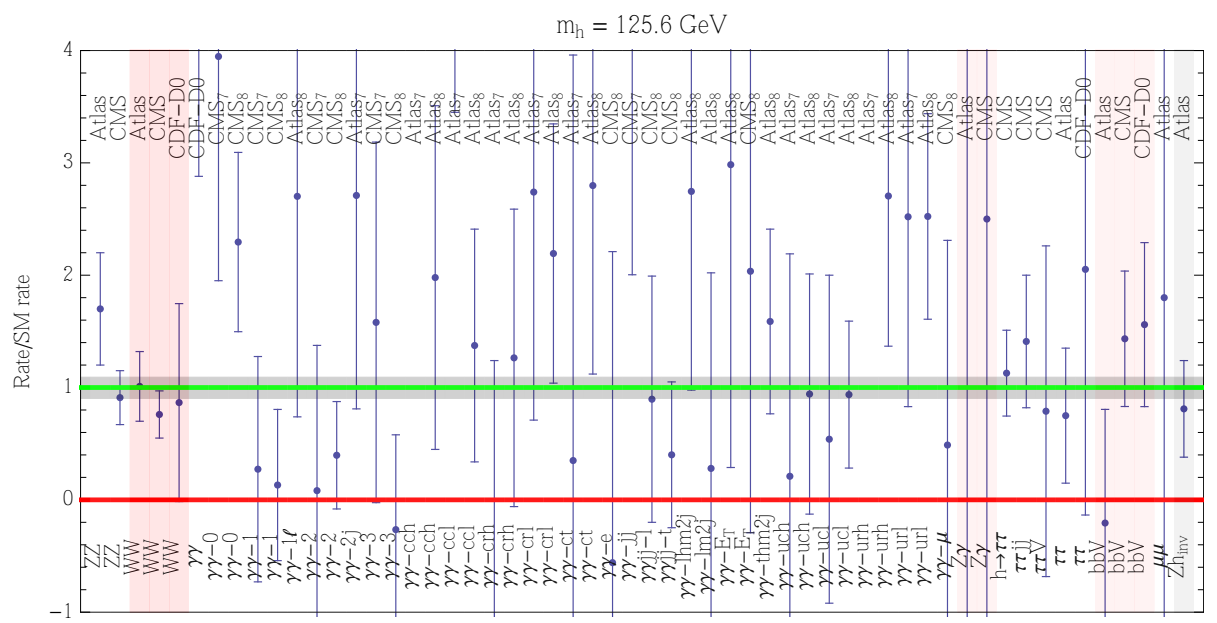

Figure 1: Measured Higgs boson rates and their average (horizontal gray band at $\pm 1 \sigma$ ). Here 0 (red line) corresponds to no Higgs boson, 1 (green line) to the SM Higgs boson.

\section{Introduction}

We want to know if the particle recently found at LHC $[1,2]$ is the long-waited Standard Model (SM) Higgs boson [3]. To do that we can approximatively reconstruct from the data its production cross sections, decay modes and couplings and check if they agree with the predictions of the SM or of other BSM theories.

In particular we make the assumption that new physics can be approximated as a first-order perturbation with respect to the SM predictions. We find that this assumption is supported by data, that agree with the SM with precisions around the $20 \%$ level. For details we refer to [4].

For this analysis we used the data presented at the Moriond 2013 conference by the CMS, ATLAS and TeVatron collaborations [5, 6, 7, 8] in the following five decay modes: $\gamma \gamma$ [9], ZZ $Z^{*}[10]$, $W W^{*}[11,6], \tau^{+} \tau^{-}$[12], $b \bar{b}[7,13], \mu^{+} \mu^{-}$[14], $Z \gamma$ [15] and $W W W$ [16], as well as their combination [17]. The data are summarized together with their $1 \sigma$ error-bars in fig. 1 . The grey band shows the $\pm 1 \sigma$ range for the average of all rates: $1.00 \pm 0.10$.

From the measured Higgs boson rates $R$ in units of the central value of the SM prediction, reported by the experimental collaborations, the $\chi^{2}$ is approximated as

$$
\chi^{2}=\sum_{I} \frac{\left(R_{I}^{\exp }-1\right)^{2}}{\left(R_{I}^{\operatorname{err}}\right)^{2}}
$$

where the sum runs over all measured Higgs boson rates $I$.

\section{The universal Higgs fit}

We consider the following effective Lagrangian:

$$
\begin{aligned}
\mathscr{L}_{h}= & r_{t} \frac{m_{t}}{V} h \bar{t} t+r_{b} \frac{m_{b}}{V} h \bar{b} b+r_{\tau} \frac{m_{\tau}}{V} h \bar{\tau} \tau+r_{\mu} \frac{m_{\tau}}{V} h \bar{\mu} \mu+r_{Z} \frac{M_{Z}^{2}}{V} h Z_{\mu}^{2}+r_{W} \frac{2 M_{W}^{2}}{V} h W_{\mu}^{+} W_{\mu}^{-}+ \\
& +r_{\gamma} c_{\mathrm{SM}}^{\gamma \gamma} \frac{\alpha}{\pi V} h F_{\mu \nu} F_{\mu v}+r_{g} c_{\mathrm{SM}}^{g g} \frac{\alpha_{s}}{12 \pi V} h G_{\mu \nu}^{a} G_{\mu \nu}^{a}+r_{Z \gamma} c_{\mathrm{SM}}^{Z \gamma} \frac{\alpha}{\pi V} h F_{\mu v} Z_{\mu v} .
\end{aligned}
$$


where the couplings $h \gamma \gamma$, $h g$ and $h Z \gamma$ arise at one loop level in the SM. Furthermore, we take into account the possibility of Higgs decays into invisible particles $X$ (such as Dark Matter or neutrinos [18]) with branching ratio $\mathrm{BR}_{\text {inv }}$. Any possible new-physics model can be described as specific values of the $r_{i}$ parameters.

Following the procedure described in the previous section, we can extract from the data the function

$$
\chi^{2}\left(r_{t}, r_{b}, r_{\tau}, r_{W}, r_{Z}, r_{g}, r_{\gamma}, r_{Z \gamma}, r_{\mu}, \mathrm{BR}_{\mathrm{inv}}\right)
$$

which describes all the information contained in Higgs data. We find $\chi^{2}=58.8$ at the best fit (56 data points, 10 free parameters), marginally better than the SM fit, $\chi_{\mathrm{SM}}^{2}=61.7$ (no free parameters).

The universal $\chi^{2}$ of eq. (2.2) has a too complicated form to be reported analytically, and depends on too many variables to be reported in numerical form, like plots or tables. However, since the data are converging towards the SM predictions with small errors, it is reasonable to make the approximation

$$
r_{i}=1+\varepsilon_{i} \quad \text { with } \quad \varepsilon_{i} \ll 1
$$

and $\mathrm{BR}_{\mathrm{inv}}=\varepsilon_{\mathrm{inv}}$. The observable rates $R_{I}$ are computed at first order in $\varepsilon_{i}$, and consequently the $\chi^{2}$ is expanded up to second order in $\varepsilon_{i}$. For example, for LHC at $8 \mathrm{TeV}$ the rate of $h \rightarrow W W$ is approximated as

$$
R_{h \rightarrow W W}=1-1.14 \varepsilon_{b}+1.58 \varepsilon_{g}-\varepsilon_{\mathrm{inv}}-0.04 \varepsilon_{t}+1.72 \varepsilon_{W}+0.02 \varepsilon_{Z}-0.13 \varepsilon_{\tau},
$$

and the full $\chi^{2}$ can now be reported in a simple form:

$$
\chi^{2}=\sum_{i, j}\left(\varepsilon_{i}-\mu_{i}\right)\left(\sigma^{2}\right)_{i j}^{-1}\left(\varepsilon_{j}-\mu_{j}\right), \quad \text { where } \quad\left(\sigma^{2}\right)_{i j}=\sigma_{i} \rho_{i j} \sigma_{j}
$$

in terms of the mean values $\mu_{i}$ of each parameter $\varepsilon_{i}$, of its error $\sigma_{i}$ and in terms of the correlation matrix $\rho_{i j}$. Since the uncertainties on the $\varepsilon_{i}$ parameters are now smaller then 1 , the universal approximation starts to be accurate.

\section{Model-dependent Higgs fits}

\subsection{Standard Model}

Assuming the SM predictions for the Higgs decays, we extract from the data the Higgs production cross sections. We find that they agree with the SM predictions, as shown in fig. 2a. As expected, the most precisely probed cross section is the dominant one, $\sigma(p p \rightarrow h)$. If we now assume that only the SM particles contribute to the $h \rightarrow g g, \gamma \gamma, \gamma Z$ loops:

$$
r_{g}=r_{t}, \quad r_{\gamma} \approx 1.282 r_{W}-0.282 r_{t} \quad r_{Z \gamma} \approx 1.057 r_{W}-0.057 r_{t}
$$

we find that the fit to the Higgs boson couplings to vectors and fermions is shown in fig. $2 \mathrm{~b}$ and agrees with the SM predictions (diagonal line), 

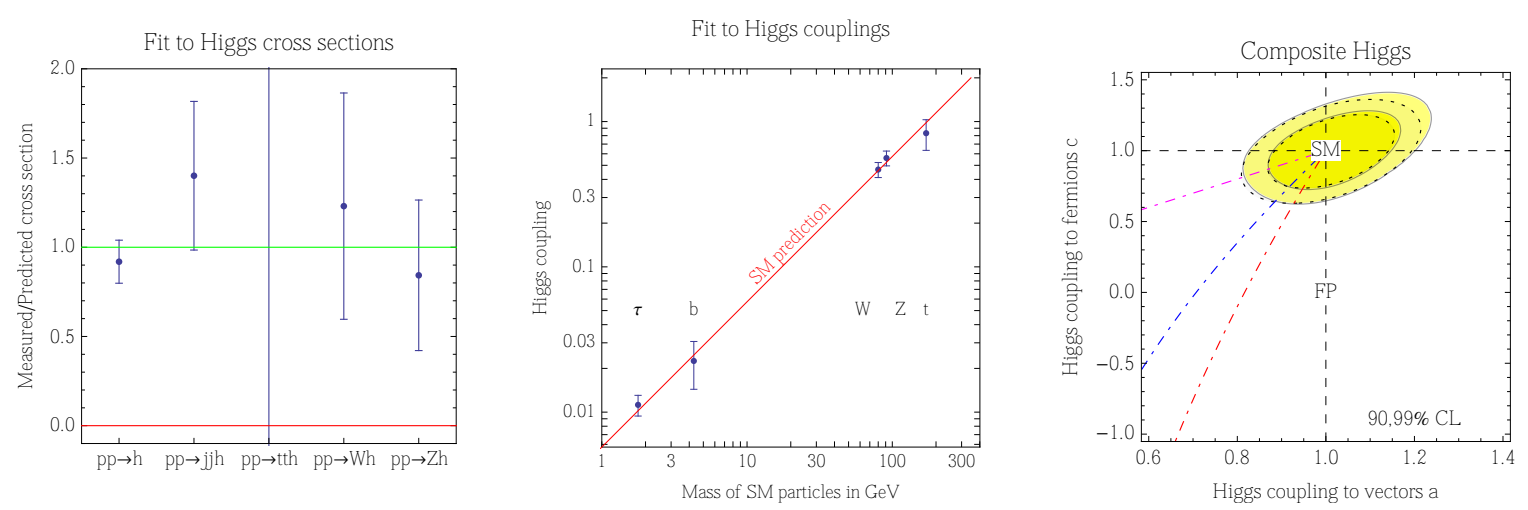

Figure 2: Left: reconstruction of the Higgs production cross sections in units of the SM prediction. Middle: reconstruction of the Higgs couplings to the $t, Z, W, b, \tau$, assuming that no new particles exist. Right:fit of the Higgs boson couplings assuming common rescaling factors a and $c$ with respect to the SM prediction. Dotted lines show the "universal" fit approximation.

\subsection{Composite Higgs models}

In a composite Higgs models, in addition to eq. (3.1), the Higgs boson couplings to massive vector bosons and fermions are rescaled:

$$
r_{t}=r_{b}=r_{\tau}=r_{\mu}=c, \quad r_{W}=r_{Z}=a
$$

The resulting fit is shown in fig. 2c. We see that our approximated universal fit (dotted contours) reproduces very well our full fit (continuous contours).

\subsection{New physics only in the loop processes}

We here assume that only the loop processes are modified with respect to the SM predictions. This amounts to restrict our universal fit setting

$$
r_{t}=r_{b}=r_{\tau}=r_{\mu}=r_{W}=r_{Z}=1, \quad \frac{\Gamma(h \leftrightarrow g g)}{\Gamma(h \leftrightarrow g g)_{\mathrm{SM}}}=r_{g}^{2}, \quad \frac{\Gamma(h \rightarrow \gamma \gamma)}{\Gamma(h \rightarrow \gamma \gamma)_{\mathrm{SM}}}=r_{\gamma}^{2}
$$

with $\mathrm{BR}_{\mathrm{inv}}=0$ and $r_{Z \gamma}=1$. The result is shown in the left panel of fig. 3, under the form of a fit to the ratios of $\mathrm{BR}(h \rightarrow g g)$ and $\mathrm{BR}(h \rightarrow \gamma \gamma)$ with respect to the $\mathrm{SM}$. The dashed trajectories show the loop effect due to extra scalar particles with the same quantum numbers of the top (red), of the bottom (blue), of the tau (vertical black line).

\subsection{Higgs boson invisible width}

Next, we allow for a Higgs boson invisible width. We perform two fits. In the first fit, the invisible Higgs width is the only new physics. We find

$$
\mathrm{BR}_{\mathrm{inv}}=-0.08 \pm 0.16 \quad \text { i.e. } \quad \mathrm{BR}_{\mathrm{inv}}<0.19 \text { at } 95 \% \text { C.L. }
$$



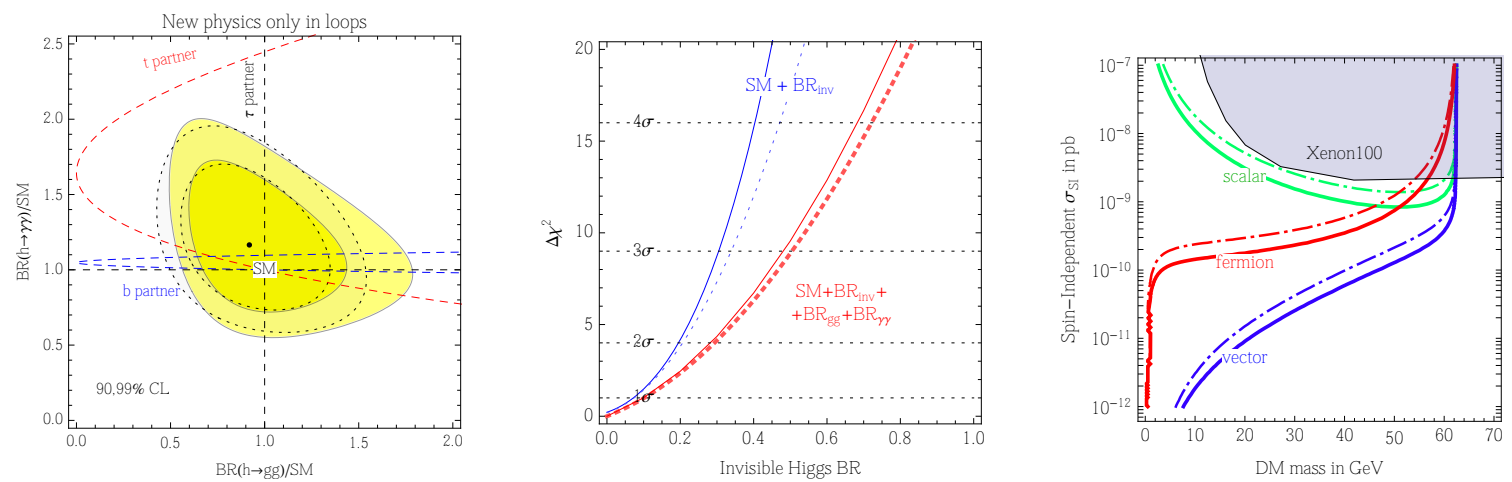

Figure 3: Left: fit for the Higgs boson branching fraction to photons and gluons, with 1 and $2 \sigma$ contours. Middle: fits to the invisible Higgs boson branching fraction under the two different assumptions. Right: upper limit on the spin-independent DM cross section on nucleons as a function of the DM mass for scalar (green), Majorana fermion (red) and vector (blue) DM.

In the second fit, in addition to the invisible width we also allow for non-standard values of $h \rightarrow \gamma \gamma$ and $h \leftrightarrow g g$, finding a weaker constraint on $\mathrm{BR}_{\text {inv }}$ (red curves in fig. $3 \mathrm{~b}$ )

$$
\mathrm{BR}_{\mathrm{inv}}<0.28 \text { at } 95 \% \text { C.L. }
$$

We can use the bounds on $\mathrm{BR}_{\mathrm{inv}}$ to constrain [19] the elastic cross section on nucleons of Dark Matter (DM) candidates with mass below $M_{h} / 2$ :

$$
\mathrm{BR}_{\mathrm{inv}} \equiv \frac{\Gamma(h \rightarrow \mathrm{DMDM})}{\Gamma_{h}^{\mathrm{SM}}+\Gamma(h \rightarrow \mathrm{DMDM})}=\frac{\sigma_{\mathrm{SI}}}{\mu \Gamma_{h}^{\mathrm{SM}}+\sigma_{\mathrm{SI}}}
$$

where $\Gamma_{h}^{\mathrm{SM}}=4.1 \mathrm{GeV}$ is the total Higgs decay width into all SM particles, that we fix to its $\mathrm{SM}$ prediction. We can now impose the upper bounds on $\mathrm{BR}_{\text {inv. }}$. In fig. 3 we show the corresponding upper limits on the spin-independent DM cross section on nucleons as a function of the DM mass.

\section{Discussion and Conclusions}

The LHC experiments reported their measurements of Higgs boson properties at the Moriond 2013 conferences, based on the full collected luminosity during 2011 and 2012. At the same time, TeVatron reported their final Higgs results. Those results will drive our understanding of particle physics, until new $13 \mathrm{TeV}$ LHC data will be available. Motivated by these results, we have performed a state-of-the-art global fit to Higgs boson data. We found that the average Higgs rate is $1.00 \pm 0.10$ in SM units, supporting the SM Higgs boson hypothesis. We studied several new physics scenarios beyond the SM. Qualitatively, all the fits reach to the same conclusions:

i) best fit regions lie along SM predictions, imposing constraints on new physics;

ii) our simple 'universal' approximation to the full fit is adequate. 


\section{Acknowledgments}

This work has been partially funded by the "Fondazione A. della Riccia".

\section{References}

[1] ATLAS Collaboration, Observation of a new particle in the search for the Standard Model Higgs boson with the ATLAS detector at the LHC, Phys. Lett. B716 (2012) 1, [hep-ex: 1207.7214$].$

[2] CMS Collaboration, Observation of a new boson at a mass of $125 \mathrm{GeV}$ with the CMS experiment at the LHC, Phys. Lett. B716 (2012) 30 [hep-ex: 1207 . 7235].

[3] F. Englert and R. Brout, Broken Symmetry and the Mass of Gauge Vector Mesons, Phys. Rev. Lett. 13 (1964) 321. P. W. Higgs, Broken symmetries, massless particles and gauge fields, Phys. Lett. 12 (1964) 132. P. W. Higgs, Broken Symmetries and the Masses of Gauge Bosons, Phys. Rev. Lett. 13 (1964) 508. G. S. Guralnik, C. R. Hagen and T. W. B. Kibble, Global Conservation Laws and Massless Particles, Phys. Rev. Lett. 13 (1964) 585.

[4] P. P. Giardino, K. Kannike, I. Masina,M. Raidal and A. Strumia, The universal Higgs fit, hep-ph:1303.3570.

[5] Guillelmo Gomez-Ceballos, CMS Collaboration, Talk at the Moriond 2013 EW session.

[6] Fabrice Hubaut, ATLAS Collaboration, Talk at the Moriond 2013 EW session. Eleni Mountricha, ATLAS Collaboration, Talk at the Moriond 2013 QCD session.

[7] Valentina Dutta, CMS Collaboration, Talk at the Moriond 2013 EW session. Victoria Martin, ATLAS Collaboration, Talk at the Moriond 2013 EW session.

[8] Lidija Živković, CDF and DO Collaborations, Talk at the Moriond 2013 EW session.

[9] CMS Collaboration, CMS-PAS-HIG-13-001. ATLAS Collaboration, ATLAS-CONF-2013-012.

[10] CMS Collaboration, CMS-PAS-HIG-13-002. ATLAS Collaboration, ATLAS-CONF-2013-013.

[11] CMS Collaboration, CMS-PAS-HIG-13-003. ATLAS Collaboration, ATLAS-CONF-2013-030.

[12] CMS Collaboration, CMS-PAS-HIG-13-004. ATLAS Collaboration, ATLAS-CONF-2012-160.

[13] ATLAS Collaboration, ATLAS-CONF-2012-161.

[14] ATLAS Collaboration, ATLAS-CONF-2013-010.

[15] CMS Collaboration, CMS-PAS-HIG-13-006. ATLAS Collaboration, ATLAS-CONF-2013-009.

[16] CMS Collaboration, CMS-PAS-HIG-13-009.

[17] Mingshui Shen, CMS Collaboration, Talk at the Moriond 2013 EW session. Bruno Mansoulie, CMS Collaboration, Talk at the Moriond 2013 EW session. ATLAS Collaboration, ATLAS-CONF-2013-014.

[18] K. Belotsky, D. Fargion, M. Khlopov, R. Konoplich and K. Shibaev, Invisible Higgs boson decay into massive neutrinos of fourth generation, Phys. Rev. D68 (2003) 054027 [hep-ph / 0210153 ].

[19] J. R. Espinosa, M. Muhlleitner, C. Grojean and M. Trott, Probing for Invisible Higgs Decays with Global Fits, JHEP 1209 (2012) 126, [hep-ph/1205 . 6790]. G. Belanger, B. Dumont, U. Ellwanger, J. F. Gunion and S. Kraml, Status of invisible Higgs decays, Phys. Lett. B723 (2013) 340, [hep-ph/1302.5694]. 